\title{
Fully Differential Cross Sections for Single Ionization of Helium by Ion Impact
}

D. Fischer ${ }^{1}$, R. Moshammer ${ }^{1}$, M. Schulz ${ }^{2}$, A. Voitkiv ${ }^{1}$, D.H. Madison ${ }^{2}$, M. Foster ${ }^{2}$, and J. Ullirich ${ }^{1}$

\footnotetext{
${ }^{1}$ Max-Planck Institut für Kernphysik, Saupfercheckweg 1, D-69117 Heidelberg, Germany

${ }^{2}$ University of Missouri-Rolla, Physics Department and Laboratory for Atomic, Molecular, and Optical Research, Rolla, Missouri 65409, USA
}

\begin{abstract}
We present experimental and theoretical fully differential cross sections for single ionization of He by heavy-ion impact for electrons emitted into the scattering plane. Data were obtained for $2 \mathrm{MeV} / \mathrm{amu} \mathrm{C}^{6+}$ and $3.6 \mathrm{MeV} / \mathrm{amu} \mathrm{Au}^{\mathrm{Q}+}(\mathrm{Q}=24,53)$ projectiles, corresponding to perturbations (projectile charge to velocity ratio) ranging from 0.7 to 4.4 , a regime which is not accessible for electron-impact ionization. We observe a decreasing recoil peak intensity (relative to the binary peak) and at the same time an increasing peak in the forward direction with increasing perturbation. Large discrepancies between experiment and theory are found, which can at least partly be attributed to the use of hydrogenic wavefunctions.
\end{abstract}




\section{Introduction}

Single ionization by charged particle impact is a particularly suitable reaction to study the fundamentally important few-body problem. The final space state of the collision (involving only unbound particles), which needs to be determined to measure fully differential cross sections, is readily obtained if the momentum vectors of all collision fragments are known. For excitation, in contrast, determination of the final electron (bound) state requires the knowledge of at least three quantum numbers ( $\mathrm{n}, \mathrm{l}$, and $\mathrm{m}_{1}$ ), which are usually difficult to obtain [1].

Since the pioneering work of Ehrhardt et al. [2], fully differential single ionization cross sections for electron impact have been measured extensively [3-8]. The vast majority of these studies were performed for electrons ionized into the scattering plane [2-7], which is defined by the initial and scattered projectile momentum vector. The characteristic structures observed for this geometry in the fully differential cross sections as a function of the electron emission angle is relatively simple, at least for large and intermediate projectile energies: a large peak is found approximately in the direction of the momentum transfer vector $\mathbf{q}$ (defined as the difference between the initial and final projectile momentum vectors) and a typically much smaller peak in the direction of $\mathbf{- q}$. The former can be explained in terms of a binary interaction between the projectile and the target electron (i.e. the target nucleus remains essentially passive) and is thus called the binary peak. The latter peak, dubbed the recoil peak, has been interpreted as a two-step process: the atomic electron first undergoes a binary collision with the projectile and is then backscattered by its own nucleus at $180^{\circ}$, so that it ends up moving in the direction of $-\mathbf{q}[9]$. 
In the theoretical description of single ionization by electron impact, tremendous progress was achieved in the last 15 years [e.g. 10-15]. At large projectile energies, typically reasonable agreement with experimental data is readily achieved with a first order treatment [14]. However, small but nevertheless systematic and significant discrepancies are found in the absolute magnitude of the recoil peak. These deviations are somewhat reduced in second order Born calculations [14] and models which account for higher order effects in the final state wavefunction $[10,13]$, but they are not completely eliminated at least for targets other than H. Overall, with such higher-order calculations satisfactory agreement with experimental data can be obtained for projectile energies as low as about twice the ionization potential. Non-perturbative methods, such as convergent close coupling (CCC) [12] and exterior complex scaling (ECS) [15] have been applied to projectile energies just a few $\mathrm{eV}$ above threshold. Impressive agreement, both qualitative and quantitative, with experimental data for an atomic hydrogen target [7], especially after revision of the measurements [16], was achieved. However, some discrepancies still remain near threshold for certain kinematic conditions [16].

For ion impact, kinematically complete experiments on single ionization are significantly more challenging than for electron impact because, with increasing projectile mass and energy, it becomes increasingly difficult to measure the scattered projectile momentum. As a result, until recently the most detailed experiments measuring the scattered projectile momentum directly only provided doubly differential single ionization cross sections as a function of scattering angle and electron energy [17,18]. The first kinematically complete experiment on single ionization involving a direct measurement of 
the scattered projectile momentum for light ions at intermediate energies was reported only a couple of years ago [19].

For heavy ions at large energies, kinematically complete experiments are only possible by an indirect determination of the scattered projectile momentum. To this end, the development of Cold Target Recoil Ion Momentum Spectroscopy (COLTRIMS) represented a crucial break-through [20,21]. When this method was later refined to include an electron detector, it became possible to measure the momentum vectors of the ionized electron and the recoiling target ion directly and thus to deduce the scattered projectile momentum from momentum conservation. The first kinematically complete experiment using this technique was reported in 1994 [22]. However, it took another 7 years before the first fully differential cross sections for single ionization by ion impact were reported [23].

For small perturbation $\mathrm{Q}_{\mathrm{p}} / \mathrm{v}_{\mathrm{p}}$ (where $\mathrm{Q}_{\mathrm{p}}$ and $\mathrm{v}_{\mathrm{p}}$ are the projectile charge and velocity, respectively) the data for the scattering plane, as expected, were found to be consistent with those for electron impact under otherwise similar conditions [4]. Furthermore, the experimental cross sections were very well reproduced not only by a sophisticated Continuum Distorted Wave - Hartree-Fock (CDW-HF) calculation, but even by the first Born approximation [24]. It was therefore very surprising when qualitative and large discrepancies were found for the cross sections outside the scattering plane [25]. This work showed that, even for small perturbation, ionization is not as well understood as was previously assumed based on studies for the scattering plane.

For large perturbations, the theoretical description of ionization is significantly more challenging than for small perturbations. One important advantage of studies on ionization by ion impact is that the perturbation can be increased practically without limitation by 
increasing the projectile charge. For electron impact ionization of $\mathrm{He}$, in contrast, the maximum perturbation that can be reached is 0.7 . Recently, we reported fully differential cross sections for $3.6 \mathrm{MeV} / \mathrm{amu} \mathrm{Au}^{53+}+\mathrm{He}$ collisions corresponding to a perturbation of 4.4 [26]. An important observation in that work was the complete absence of a peak in the direction of $\mathbf{- q}$ (where the recoil peak is observed for small perturbations). Instead, a pronounced maximum was found in the forward direction which for small momentum transfers was the dominant feature in the data. A continuum distorted wave - eikonal initial state (CDW-EIS) calculation [27], in contrast, showed a binary peak as the only structure in the fully differential cross sections.

A possible explanation, that was discussed in the same work [26], is that the maximum in the forward direction is actually the recoil peak which, however, is strongly shifted due to the post-collision interaction (PCI) between the outgoing projectile and the ionized electron. It was further argued that in the CDW-EIS calculation, the PCI leads to a nonorthogonality between the initial and final state electron wavefunctions. This nonorthogonality, in turn, might lead to an artificial monopole term, which could suppress the recoil peak, an effect well-known for the plane wave Born approximation [28]. In this work, we investigate this explanation in more detail by systematically studying the fully differential single ionization cross sections as a function of the perturbation.

\section{Experiment}

The final state of an ion-atom collision system after single ionization involves 3 unbound particles (the electron, the projectile, and the recoiling target ion). Thus, a kinematically complete experiment requires measuring the momentum vectors of 2 particles. The third 
momentum vector can then be deduced from momentum conservation. In the experiments described below, the momentum vectors of the ionized electron and the recoil ion were measured.

The experiments were performed at the Gesellschaft für Schwerionenforschung (GSI) in Darmstadt and the Max-Planck Institut für Kernphysik (MPI-K) in Heidelberg. For the experiments at GSI 3.6 MeV/amu $\mathrm{Au}^{\mathrm{Q}+}(\mathrm{Q}=24,53)$ beams were delivered by the Universal Linear Accelerator (UNILAC) and collimated to a size of about $1 \times 1 \mathrm{~mm}^{2}$. At MPI-K, a 2 $\mathrm{MeV} / \mathrm{amu} \mathrm{C}^{6+}$ beam was obtained from the MP tandem accelerator. The projectile beams were then crossed with an atomic He beam from a supersonic gas jet. A switching magnet was used to analyze the projectile charge state after the collision and the projectiles which did not change charge state were detected by a scintillator.

The two stage supersonic gas jet is required to cool the target gas to minimize the momentum spread of the recoil ions due to their thermal motion. Helium gas can escape from a reservoir at about 15 atm through a $30 \mu \mathrm{m}$ nozzle into the first stage, which is kept at a vacuum of about 10 mTorr by a roots pump. The large pressure gradient leads to adiabatic expansion, which results in a cooling of the gas in the direction of the gradient to a temperature of less than 1 Kelvin. In the plane perpendicular to the pressure gradient, the gas is geometrically cooled by a skimmer with a diameter of $300 \mu \mathrm{m}$, which collimates those He atoms out which have a non-zero momentum component in that plane. In the second stage, which is separated from the first stage by the skimmer, a vacuum of $10^{-5}$ Torr is maintained by a $400 \mathrm{l} / \mathrm{s}$ turbomolecular pump. A second skimmer with a diameter of 600 $\mu \mathrm{m}$ separates the collision chamber from the second stage of the jet. At the intersection 
point with the projectile beam, the gas jet has a diameter of about $1 \mathrm{~mm}$ and a thickness of $10^{11} / \mathrm{cm}^{2}$. With the full gas load in the reservoir of the gas jet, the vacuum in the collision chamber was in the $10^{-8}$ Torr range.

The electrons and recoil ions were momentum-analyzed by the same spectrometer system. It consists of two parallel resistive plates $22 \mathrm{~cm}$ in length which are oriented along the projectile beam axis and separated by a distance of $7 \mathrm{~cm}$. An electric field was generated by applying a voltage of $30 \mathrm{~V}\left(60 \mathrm{~V}\right.$ for the $\mathrm{C}^{6+}$ projectiles $)$ across the plates so that the electrons were extracted parallel and the recoil ions anti-parallel to the projectile beam direction. After traversing a $22 \mathrm{~cm}$ long field free drift tube following the extraction region, the recoil ions and electrons were detected by two position-sensitive channel plate detectors with diameters of 50 and $80 \mathrm{~mm}$, respectively.

The extraction field was not strong enough to guide a sufficiently large fraction of the electrons onto the detector. Therefore, a uniform magnetic field of $20 \mathrm{G}$ (6 G for the $\mathrm{C}^{6+}$ projectiles) in the same direction as the electric extraction field was generated by two Helmholtz coils. As a result, the electrons were forced into cyclotron motion with a radius proportional to the transverse momentum component of the electrons. For transverse momenta of less than 3.5 a.u. ( 1 a.u. for the $\mathrm{C}^{6+}$ projectiles) the cyclotron radius was small enough for the electrons to hit the detector.

The electron detector was set in coincidence with both the recoil ion detector and the projectile detector. From the coincidence the time of flight of the recoil ions and the ionized electrons were obtained with a resolution of better than 1 nsec. From the time of flight, in turn, the momentum component in the longitudinal direction was determined. The two transverse components were deduced from the position information. 
The achieved momentum resolution for the recoil ions was $\Delta \mathrm{p}_{\mathrm{rl}}=0.1$ a.u. in the longitudinal direction and $\Delta \mathrm{p}_{\mathrm{rt} \perp}=0.2$ a.u. in the transverse direction. The electron resolution was estimated to be $\Delta \mathrm{p}_{\mathrm{el}}=0.05$ a.u. and $\Delta \mathrm{p}_{\mathrm{et} \perp}<0.1$ a.u., respectively. Due to the cyclotron motion, however, the actual electron transverse momentum resolution depends on the magnitude and direction of the initial electron momentum vector. The transverse momentum transfer of the projectile is calculated event by event from the transverse momenta of the ejected electron and the recoil ion $\mathbf{q}_{\mathbf{t} \perp}=\left(\mathbf{p}_{\mathrm{et} \perp}+\mathbf{p}_{\mathrm{rt} \perp}\right)$ making use of momentum conservation. The total momentum transfer is given by $\mathbf{q}=\mathbf{q}_{\mathbf{t} \perp}+\mathbf{q}_{\min } \cdot \hat{\mathbf{v}}_{\mathrm{p}}$, where $\hat{\mathrm{v}}_{\mathrm{p}}$ is the unit vector along the initial projectile velocity with $\hat{\mathrm{v}}_{\mathrm{p}} \cdot \mathbf{q}_{\mathbf{t} \perp}=0$. The quantity $\mathrm{q}_{\min }=\left(\mathrm{I}+\mathrm{E}_{\mathrm{e}}\right) / \mathrm{v}_{\mathrm{p}}$ is the minimum momentum transfer (corresponding to zero degree scattering) required to overcome the binding energy I and to promote the electron into a continuum state with energy $\mathrm{E}_{\mathrm{e}}$. For the present projectiles and for low energy electron emission $\left(\mathrm{E}_{\mathrm{e}}<15 \mathrm{eV}\right)$ the longitudinal momentum transfer is very small $\mathrm{q}_{\min }<0.2$ a.u. and it can be deduced with very high accuracy directly from the measured electron energy. The uncertainty in the determination of $\mathrm{q}_{\perp}$, which amounts to $\Delta \mathrm{q}_{\perp}<0.22$ a.u., is related to the achieved transverse momentum resolution for the recoil ion and the electron. The sums of all recorded events are normalized to the theoretical (CDW-EIS calculation) total He single ionization cross sections of $\sigma_{6+}=3.8 \cdot 10^{-16} \mathrm{~cm}^{2}, \sigma_{24+}=2.7 \cdot 10^{-15} \mathrm{~cm}^{2}$ and $\sigma_{53+}=8.3 \cdot 10^{-15}$ $\mathrm{cm}^{2}$ for $2 \mathrm{MeV} / \mathrm{amu} \mathrm{C}^{6+}$ and $3.6 \mathrm{MeV} / \mathrm{amu} \mathrm{Au}^{\mathrm{Q}+}$ projectiles, respectively. 


\section{Results and discussion}

From the kinematically complete data, we obtained fully differential single ionization cross sections $\mathrm{d}^{5} \sigma /\left(\mathrm{d}^{2} \mathbf{q}_{\perp} \mathrm{d}^{3} \mathbf{k}\right)$, where $\mathbf{q}_{\perp}$ is the transverse momentum transfer and $\mathbf{k}$ is the final state electron momentum vector. These cross sections are more commonly referred to as triply differential cross sections (TDCS) because $\mathrm{d}^{2} \mathbf{q} \mathrm{d}^{3} \mathbf{k}$ can be expressed in terms of the triple differential $\mathrm{d} \Omega_{\mathrm{p}} \mathrm{d} \Omega_{\mathrm{e}} \mathrm{dE}$ (where $\Omega_{\mathrm{p}}$ and $\Omega_{\mathrm{e}}$ are the solid angles for the projectile and the electron and $E_{e}$ is the electron energy). Here, we present the TDCS for electrons emitted into the scattering plane. Earlier, we reported these TDCS for the case of the 3.6 $\mathrm{MeV} / \mathrm{amu} \mathrm{Au}^{53+}$ projectiles for momentum transfers of 0.65 a.u. and larger and for electron energies of $17.5 \mathrm{eV}$ and larger [26]. As mentioned above, in that work we found that the maximum in the forward direction seemed to increase (relative to the binary peak) with decreasing momentum transfer and electron energy. Here, we therefore focus on the momentum transfer and electron energy dependence for relatively small values of both quantities. In addition, the evolution of the TDCS from an intermediate perturbation $\left(\mathrm{Q}_{\mathrm{p}} / \mathrm{v}_{\mathrm{p}}\right.$ $=0.7$ for $2 \mathrm{MeV} / \mathrm{amu} \mathrm{C}^{6+}$ projectiles) up to the highly non-perturbative regime $\left(\mathrm{Q}_{\mathrm{p}} / \mathrm{v}_{\mathrm{p}}=2.0\right.$, 4.4 for the $\mathrm{Au}^{\mathrm{Q}+}$ projectiles) is presented.

The experimental data are compared with three different calculations (Figs. 1-3). The dotted lines show the first Born approximation (FBA) and results of CDW-EIS calculations are shown without (dashed curves) and with (solid curves) the projectile - target nucleus interaction included (CDWNN-EIS). Single ionization of He is regarded as an effective single electron process in all models. In the initial and final states the active electron is assumed to move in the Coulomb field of the target with an effective charge of $Z_{t e}=1.34$. 
The cross sections calculated with this hydrogen-like description are multiplied by a factor of two to account for the second target electron. The Coulomb interaction between the projectile and the electron is considered within the CDW-EIS approach [29]. The projectile - target nucleus interaction is treated (CDWNN-EIS model) as a pure Coulomb interaction between the projectile and the target core with a net charge of $\mathrm{Z}_{\mathrm{tp}}=1$. Here, the distortion due to the projectile - target nucleus interaction is accounted for by an eikonal factor, representing the asymptotic of the corresponding two-body Coulomb wave, both in the initial and in the final channel [30]. Such an approximation is quite reasonable for small projectile deflections and negligible recoil ion velocities compared to that of the emitted electron. Both conditions are very well fulfilled for the present cases.

In Fig. 1 the fully differential cross sections are shown for $2 \mathrm{MeV} / \mathrm{amu} \mathrm{C}^{6+}+\mathrm{He}$ for electrons emitted into the scattering plane with energies $E_{e}$ of $4 \mathrm{eV}$ (part a) and $10 \mathrm{eV}$ (part b) and momentum transfers of $0.45,0.65$, and 1.0 a.u. We observe a clear recoil peak which is well separated from the binary peak. In fact, for large momentum transfers the data look quite similar to what is typically observed for small perturbations [4,23]. All calculations shown in Fig. 1 are divided by a factor of 2 to better illustrate the comparison in shape with the data. There is a nearly uniform discrepancy in magnitude of a factor of 2 between the CDWNN-EIS calculation and the data, while for both the FBA and the CDWEIS models the agreement in magnitude improves with decreasing q. In the shape, already for the present perturbation of 0.7 systematic deviations between experiment and theory appear. For example, the intensity ratios between the binary and recoil peaks are overestimated by all models. This effect is particularly pronounced for the CDWNN-EIS model, which grossly overestimates that ratio for all kinematic conditions. In addition, the 
considerable contribution in the forward direction, more distinct for small momentum transfers and small electron energies, is not reproduced by the theoretical approaches considered here.

In Figs. 2 and 3 we show the same cross sections for the same kinematic conditions as in Fig. 1 for $3.6 \mathrm{MeV} / \mathrm{amu} \mathrm{Au}^{24+}$ and $3.6 \mathrm{MeV} / \mathrm{amu} \mathrm{Au}^{53+}$ colliding with He. Compared to the $\mathrm{C}^{6+}$ results significant changes are observed while the data for both charge states of the Au projectiles look qualitatively similar. In both cases we find strong contributions in the forward direction and no peak structures near the direction of $\mathbf{- q}$. The binary lobes of the FBA results (dotted lines), which are symmetric with respect to the momentum transfer, indicate the direction of $\mathbf{q}$ in each case. For the $\mathrm{Au}^{24+}$ projectiles, the TDCS is dominated by two contributions: a forward shifted binary peak, which appears to be the largest structure for large momentum transfers (1.0 a.u.), and a generally weaker but distinct peak in the forward direction. The weighting of these two contributions is reversed in the data for the $\mathrm{Au}^{53+}$ projectiles. There the general trend observed in the data is consistent with the results reported earlier for the same collision system [26]: with decreasing q the cross sections are increasingly dominated by a peak in the forward direction. In fact, only for the largest momentum transfer (1.0 a.u.) and only at $\mathrm{E}_{\mathrm{e}}=10 \mathrm{eV}$ can a clear peak structure near the direction of $\mathbf{q}$ be discerned. Under no kinematic condition do we ever observe a peak near the direction of $\mathbf{- q}$.

At the large perturbations of 2.0 and 4.4 corresponding to the $\mathrm{Au}^{24+}$ and $\mathrm{Au}^{53+}$ projectiles, respectively, the first Born approximation is not expected to provide a good description of the ionization process. Only for completeness we show the FBA results in Figs. 2 and 3. In contrast to the FBA, CDW-EIS without the projectile - target nucleus 
interaction has been shown to describe both, total cross sections and differential electron spectra very well [31] even for large perturbations [32]. However, it fails completely to reproduce the fully differential cross sections in this regime (Figs. 2 and 3). Already the absolute values of the TDCS are off by orders of magnitudes for certain kinematical conditions. One would expect the CDW-EIS model with the projectile - target nucleus interaction (CDWNN-EIS) to provide the best agreement with the current data. Instead, we observe that the CDWNN-EIS model does an even worse job in describing certain aspects of the data than both the CDW-EIS and the FBA calculations. The surprising fact, that in some cases the CDWNN-EIS predicts the binary peak to be shifted counter-intuitively into the backward direction for the case of $\mathrm{Au}^{53+}$ projectiles rises the question about the general validity of the CDW-EIS approach at these high perturbations. This break-down of CDWEIS calculations has already been observed previously where the single ionization cross section differential in the momentum transfer and the electron emission energy was investigated for the same collision system [33]. There, it was found that the differential cross sections calculated with the CDWNN-EIS model strongly depend on the choice of the interaction potential between the projectile and the ionic core of the target. The inclusion of the passive electron of the He target, effectively screening the target nucleus, turned out to be important. This effect is not considered in the present theoretical models. Thus, part of the discrepancies between experiment and theory might be due to the restrictions imposed by using hydrogenic wavefunctions in the calculations.

To show the dependence of the cross sections on the perturbation in a more systematic manner, in Fig. 4 we present the data for the three collisions systems for fixed electron energy and momentum transfer in order of increasing perturbation (from left to right). The 
plots in the top are for $\mathrm{E}_{\mathrm{e}}=4 \mathrm{eV}$ and $\mathrm{q}=0.45$ a.u. and the plots in the bottom for $\mathrm{E}_{\mathrm{e}}=10$ $\mathrm{eV}$ and $\mathrm{q}=1.0$ a.u. It is quite obvious that the zero-degree contributions strongly increase while at the same time the recoil peak decreases with increasing perturbation. Furthermore, we note that for the $\mathrm{C}^{6+}$ projectiles at small $\mathrm{q}$ and $\mathrm{E}_{\mathrm{e}}$ (top left part of Fig. 4) we observe pronounced zero-degree contributions simultaneously with a well separated recoil peak. Our initial attempt, to explain the zero-degree peak reported earlier for the $3.6 \mathrm{MeV} / \mathrm{amu}$ $\mathrm{Au}^{53+}$ projectiles [26] as a recoil peak, which is strongly shifted by the PCI, is therefore not supported by the current data. Instead, it must now be concluded that it actually represents a third peak probably unrelated to either the binary or the recoil peak.

Nevertheless, it still seems plausible that the forward peak is caused by the PCI. After all, it is not surprising, and it is indeed well established, that this attractive interaction drags the electrons along in the forward direction [34,35]. Furthermore, the dependence of the zero-degree peak on the momentum transfer (see Figs. 1-3) is consistent with an increasing importance of PCI-effects with decreasing momentum transfer observed earlier $[17,18]$. It was also thought that such effects should become increasingly important as the emitted electron velocity approaches the projectile velocity [36]. We therefore present in Fig. 5 the fully differential cross sections for the $\mathrm{C}^{6+}$ projectiles at a fixed momentum transfer of 0.45 a.u. for different electron energies (from left to right 1,4 , and $10 \mathrm{eV}$ ). The energy of an electron moving at the projectile velocity would be around $1.1 \mathrm{keV}$. The data of Fig. 5 suggest that the importance of the zero-degree contributions relative to the binary peak maximizes somewhere between 1 and $10 \mathrm{eV}$.

At first glance, this observation seems to be in conflict with what is expected for the PCI. However, it should be noted that the electron energies investigated here are very far 
from the energy of electrons moving at the projectile velocity. Our data do not rule out that the importance of the zero-degree contributions may increase again as the electron velocity approaches the projectile velocity. For small electron energies, there is another type of PCI which becomes important, one that is acting between the residual recoil ion and the ejected electron [32,37]. This recoil-ion PCI is expected to increase as the electron velocity approaches the recoil ion velocity (which is essentially zero), i.e. it follows the opposite trend as the projectile PCI. The two-center potential generated by the projectile and the residual recoil ion has a saddle which moves at a speed $\mathrm{v}_{\mathrm{s}}=\mathrm{v}_{\mathrm{o}} 1 /\left(1+\left(\mathrm{Q}_{\mathrm{p}} / \mathrm{Q}_{\mathrm{R}}\right)^{1 / 2}\right)\left(\right.$ where $\mathrm{v}_{\mathrm{o}}$ is the projectile speed and $Q_{p}$ and $Q_{R}$ are the electric charge of the projectile and the recoil ion, respectively) [38]. One may argue that for an electron speed $v_{e}$ smaller than $v_{s}$ the PCI with the recoil ion is more important, while for $\mathrm{v}_{\mathrm{e}}>\mathrm{v}_{\mathrm{s}}$ the PCI with the projectile is more important. For $2 \mathrm{MeV} / \mathrm{amu} \mathrm{C}^{6+}+\mathrm{He}$, the energy of electrons moving at a speed $\mathrm{v}_{\mathrm{e}}=\mathrm{v}_{\mathrm{s}}$ is $92 \mathrm{eV}$. The observed maximum in the relative importance of the zero-degree contributions at an energy between 1 and $10 \mathrm{eV}$ may therefore be due to an interplay between the projectile- and the recoil-ion PCI. The projectile PCI is strong enough to force the electrons in the forward direction, but the recoil-ion PCI counteracts the tendency of the projectile PCI to accelerate the electrons towards the projectile speed.

The above discussed electronic final state interactions are, at least to some extend, included in the CDW calculations. However, the overall unsatisfying agreement with the experimental data indicates that certain features of the underlying three particle dynamics are not described properly by theory. In this respect the CDWNN-EIS model should be superior to the other approaches, because there all mutual interactions are considered. Instead, by the comparison between experiment and theory (Figs. 1-3), one may be tempted 
to discard the CDWNN-EIS model. However, it would be premature to question the basic approaches of this model. Besides the already mentioned difficulties associated with the modeling of the projectile - target core interaction, a large fraction of the problems may actually be due to another aspect related to the inadequacy of the hydrogenic wavefunctions that are used. For small perturbation, it has been demonstrated that the absolute magnitudes of the cross sections calculated in the first Born approximation are very sensitive to the choice of the electron wavefunction, but the shape is rather insensitive [24]. For the much larger perturbations studied here, in contrast, the binary to recoil peak intensity ratio is strongly affected by the choice of the wavefunction. This is illustrated in Fig. 6, where we compare two different first Born calculations for $\mathrm{E}_{\mathrm{e}}=1 \mathrm{eV}$ and $\mathrm{q}=1.5$ a.u. (a) and for $\mathrm{E}_{\mathrm{e}}=4 \mathrm{eV}$ and $q=0.65$ a.u. (b). For the dashed curve, hydrogenic wavefunctions with $Z_{\text {eff }}=1.34$ were used and for the solid curve Hartree-Fock wavefunctions were used. Large differences between these two calculations are obvious: the hydrogenic wavefunctions result in a significantly larger binary to recoil peak intensity ratio compared to the Hartree-Fock wavefunctions, especially at the larger momentum transfer.

The comparison of Fig. 6 shows that the good description of the binary to recoil peak intensity ratio at small $\mathrm{q}$ by the FBA calculation is fortuitous. There are two major problems with the FBA used here. First, obviously higher-order contributions are completely ignored and second, inaccurate wavefunctions are used. It is well known that for ion impact the omission of higher-order contributions tend to decrease the binary to recoil peak intensity ratio [24]. The calculations presented in Fig. 6 show that the use of hydrogen-like wavefunctions tends to increase that ratio compared to Hartree-Fock wavefunctions. Apparently, these two problems to a large extent neutralize each other. It 
is reasonable to assume that, in the CDWNN-EIS model, the use of Hartree-Fock wavefunctions would decrease the binary to recoil peak intensity ratio as well thereby leading to an improved agreement with the experimental data.

\section{Conclusions}

We have presented a systematic study of fully differential single ionization cross sections for electrons emitted into the scattering plane for a broad range of perturbations. We find a gradual disappearance of the recoil peak accompanied by a build-up of contributions in the forward direction with increasing perturbation. At relatively small perturbation, these forward contributions are clearly separated from the recoil peak and at large perturbations, the forward contributions are distinguishable from the binary peak. We are therefore led to conclude that these contributions represent a third peak which has not been observed in the past. All previous experiments studying fully differential single ionization cross sections (both for electron and ion impact) were carried out at perturbations much smaller than those where we observe this third peak structure.

The theoretical description of fully differential single ionization cross sections proves very difficult. Even sophisticated CDW-EIS models (with or without projectile - target nucleus interaction) reveal serious discrepancies with the experimental data. For the first Born approximation, we found that the theoretical cross sections are very sensitive to the choice of the electron wavefunction and we assume that this is also the case for CDW-EIS models. To investigate which single ionization mechanisms lead to which features in the data, it is therefore important to compare to calculations using Hartree-Fock or even correlated many-electron wavefunctions. Furthermore, the use of Hartree-Fock 
wavefunctions is very important in order to investigate other potential problems with the current status of the CDW-EIS approach. For example, it is known that at large perturbation CDW-EIS calculations can yield impact parameter dependent ionization probabilities (per electron) of larger than 1 [39]. Nevertheless, even at this stage it seems rather obvious that the forward peak is closely related to the PCI between the outgoing projectile and the ionized electron.

\section{Acknowledgements}

One of us (M.S.) is grateful for the hospitality of the Max-Planck-Institut für Kernphysik in Heidelberg. This work was supported by the Leibniz-Programm of the Deutsche Forschungsgemeinschaft and the National Science Foundation under grants PHY0097902 and PHY-0070872. Valuable discussions with Drs B. Najjari and T. Kirchner are acknowledged. 


\section{Figure Captions}

Fig. 1: Fully differential cross sections $d^{5} \sigma /\left(d^{2} q_{\perp} d^{3} k\right)$ for electrons emitted into the scattering plane (TDCS) for ionization of He by $2.0 \mathrm{MeV} / \mathrm{amu} \mathrm{C}^{6+}$ projectiles. The cross sections are given in atomic units and the magnitude of the electron momentum vector $\mathrm{k}$ is fixed corresponding to electron energies $\mathrm{E}_{\mathrm{e}}$ of $4 \mathrm{eV}$ (part a) and $10 \mathrm{eV}$ (part b). The total momentum transfers $\mathbf{q}$ are $0.45,0.65$, and 1.0 a.u. Dotted lines: FBA result. Dashed lines: CDW-EIS results. Solid lines: CDWNN-EIS results. All calculations are divided by a factor of 2 .

Fig. 2: Same as Fig. 2 for $3.6 \mathrm{MeV} / \mathrm{amu} \mathrm{Au}^{24+}+\mathrm{He}$ collisions.

Fig. 3: Same as Fig. 2 for $3.6 \mathrm{MeV} / \mathrm{amu} \mathrm{Au}^{53+}+\mathrm{He}$ collisions.

Fig. 4: TDCS for fixed electron energies and momentum transfers of $E_{e}=4 \mathrm{eV}$ and $q=0.45$ a.u. (top) and $\mathrm{E}_{\mathrm{e}}=10 \mathrm{eV}$ and $\mathrm{q}=1.0$ a.u. (bottom) in order of increasing perturbation (from left to right). The arrows indicate the direction of $\mathbf{q}$ in each case.

Fig. 5: TDCS for $2 \mathrm{MeV} / \mathrm{amu} \mathrm{C}^{6+}+\mathrm{He}$ for a fixed momentum transfer of 0.45 a.u. for different electron energies (from left to right 1,4 , and $10 \mathrm{eV}$ ). The arrows indicate the direction of $\mathbf{q}$ in each case. 
Fig. 6: TDCS for $2 \mathrm{MeV} / \mathrm{amu} \mathrm{C}^{6+}+\mathrm{He}$ for a momentum transfer of 1.5 a.u. and an electron energy of $1 \mathrm{eV}$ (a) and for a momentum transfer of 0.65 a.u. and an electron energy of $4 \mathrm{eV}$ (b) calculated in the first Born approximation. For the dashed curve hyddrogenic wavefunctions and for the solid curve Hartree-Fock wavefunctions were used. 


\section{References}

[1] Williams J., J. Phys. B14, 1197 (1981)

[2] Ehrhardt H., Schulz M., Tekaat T., and Willmann K., Phys. Rev. Lett. 22, 89 (1969)

[3] Ehrhardt H., Jung K., Knoth G., and Schlemmer P., Z. Phys. D1, 3 (1986) and references therein

[4] Stefani G., Avaldi L., and Camilloni R., J. Phys. B23, L227 (1990)

[5] Lahmam-Bennani A., J. Phys. B24, 2401 (1991)

[6] Röder J., Ehrhardt H., Bray I., Fursa D.V., McCarthy I.E., J. Phys. B29, 2103 (1996)

[7] Röder J., Ehrhardt H., Pan C., Starace A.F., Bray I., and Fursa D.V., Phys. Rev. Lett. 79, $1666(1997)$

[8] Murray A.J., Woolf M.B.J., and Read F.H., J. Phys. B25, 3021 (1992)

[9] Whelan C.T., Allan R.J., Walters H.R.J., and Zhang X., in Whelan C.T., Walters H.R.J., Lahmam-Bennani A., and Ehrhardt H. (editors), (e,2e) \& related processes, Kluwer, Dordrecht, Series C: Mathematical and Physical Sciences - 414, 1 (1993)

[10] Brauner M., Briggs J.S., and Klar H., J. Phys. B22, 2265 (1989)

[11] Pindzola M.S. and Robicheaux F., Phys. Rev. A54, 2142 (1996)

[12] Bray I., Fursa D.V., Röder J., and Ehrhardt H., Phys. Rev. A57, 3161 (1998)

[13] Jones S., and Madison D.H., Phys. Rev. Lett. 81, 2886 (1998)

[14] Marchalant P., Whelan C.T., and Walters H.R.J., J. Phys. B31, 1141 (1998)

[15] Rescigno T.N., Baertschy M., Isaacs W.A., and McCurdy C.W., Science 286, 2474 (1999)

[16] J. Röder, M. Baertschy, and I. Bray, Phys. Rev. A67, 010702(R) (2003)

[17] Vajnai T, Gaus A D, Brand J A, Htwe W, Madison D H, Olson R E, Peacher J L and 
Schulz M 1995, Phys. Rev. Lett. 74, 3588

[18] Schulz M, Vajnai T, Gaus A D, Htwe W, Madison D H and Olson R E 1996, Phys. Rev. A54, 2951

[19] An L, Khayyat Kh and Schulz M 2001, Phys. Rev. A63, 030703(R)

[20] Dörner R., Mergel V., Ali R., Buck U., Cocke C.L., Froschauer K., Jagutzki O., Lencinas S., Meyerhof W.E., Nüttgens S., Olson R.E., Schmidt-Böcking H., L. Spielberger, Tökesi K., Ullrich J., Unverzagt M., and Wu W., Phys. Rev. Lett. 72, 3166-3169 (1994)

[21] Ullrich J. et al., Comments At. Mol. Phys. 30, 285 (1994)

[22] Moshammer R, Ullrich J, Unverzagt M, Schmitt W, Jardin P, Olson R E, Mann R, Dörner R, Mergel V, Buck U and Schmidt-Böcking H 1994, Phys. Rev. Lett. 73, 3371

[23] Schulz M, Moshammer R, Madison D H, Olson R E, Marchalant P, Whelan C T, Walters H R J, Jones S, Foster M, Kollmus H, Cassimi A and Ullrich J 2001, J. Phys. B34, L305

[24] Madison D., Schulz M., Jones S., Foster M., Moshammer R. and Ullrich J., J. Phys. B35, 3297 (2002)

[25] Schulz M., Moshammer R., Fischer D., Kollmus H., Madison D.H., Jones S., and Ullrich J., Nature 422, 48 (2003)

[26] Schulz M., Moshammer R., Perumal A.N., and Ullrich J., J. Phys. B35, L161 (2002)

[27] Crothers D.S.F. and McCartney M., Comput. Phys. Commun. 72, 288 (1992)

[28] Glassgold A.E. and Ialongo G., Phys. Rev. 175, 151 (1968)

[29] Fainstein P.D., Ponce V.H., and Rivarola R.D., J. Phys. B24, 3091 (1991)

[30] Rodriguez V.D., J. Phys. B29, 275 (1996) 
[31] Tribedi L.C., Richard P., Wang Y.D., Lin C.D., Gulyas L., and Rudd M.E., Phys. Rev. A58, 3619 (1998)

[32] Moshammer R., Fainstein P.D., Schulz M., Schmitt W., Kollmus H., Mann R., Hagmann S., and Ullrich J., Phys. Rev. Lett. 83, 4721 (1999)

[33] Moshammer R., Perumal A., Schulz M., Rodriguez V.D., Kollmus H., Mann R., Hagmann S., and Ullrich J., Phys. Rev. Lett. 87, 223201 (2001)

[34] Crooks G.B. and Rudd M.E., Phys. Rev. Lett. 25, 1599 (1970)

[35] Moshammer R., Ullrich J., Kollmus H., Schmitt W., Unverzagt M., Schmidt-Böcking H., Wood C.J., and Olson R.E., Phys. Rev. A56, 1351 (1997).

[36] Salin A., J. Phys. B2, 631 (1969)

[37] Schmitt W., Moshammer R., O'Rourke F.S.C., Kollmus H., Sarkadi L., Mann R., Hagmann S., Olson R.E., and Ullrich J., Phys. Rev. Lett. 81, 4337 (1998)

[38] Irby V.D., Phys. Rev. A39, 54 (1989)

[39] Kirchner T., Gulyás L., Moshammer R., Schulz M., and Ullrich J., Phys. Rev. A65, 042727 (2002) 

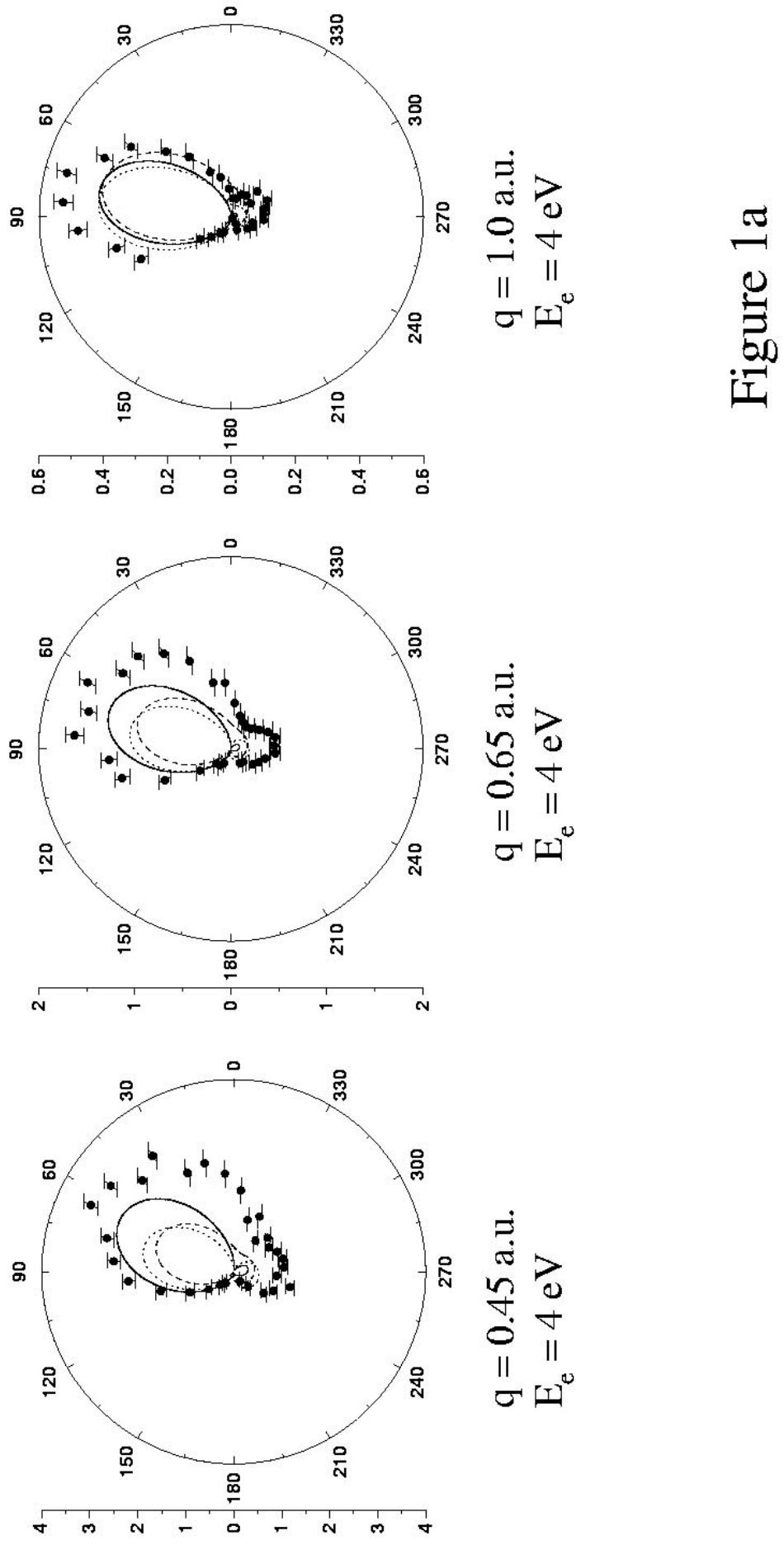

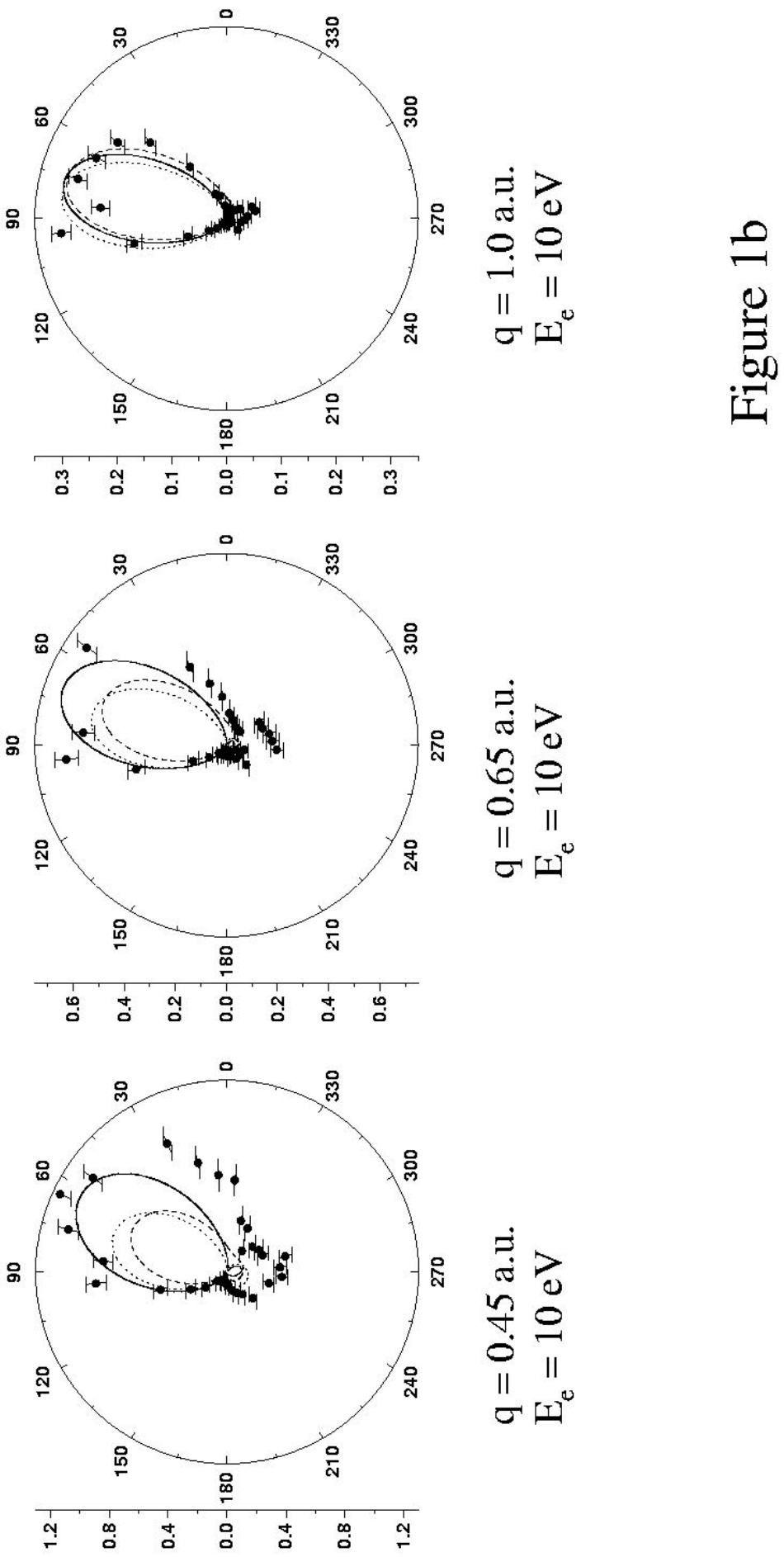

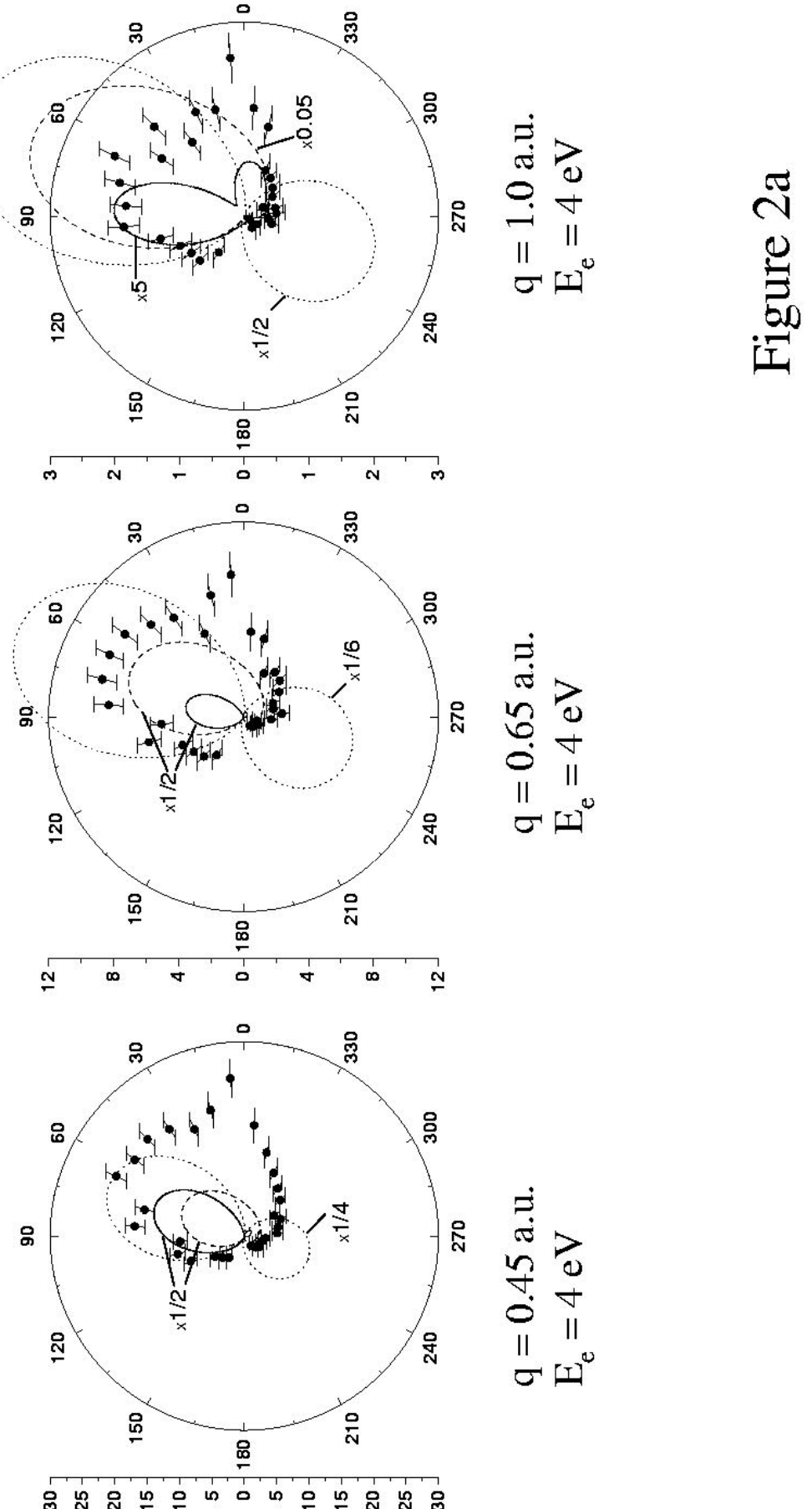

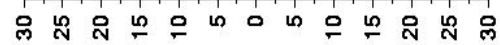



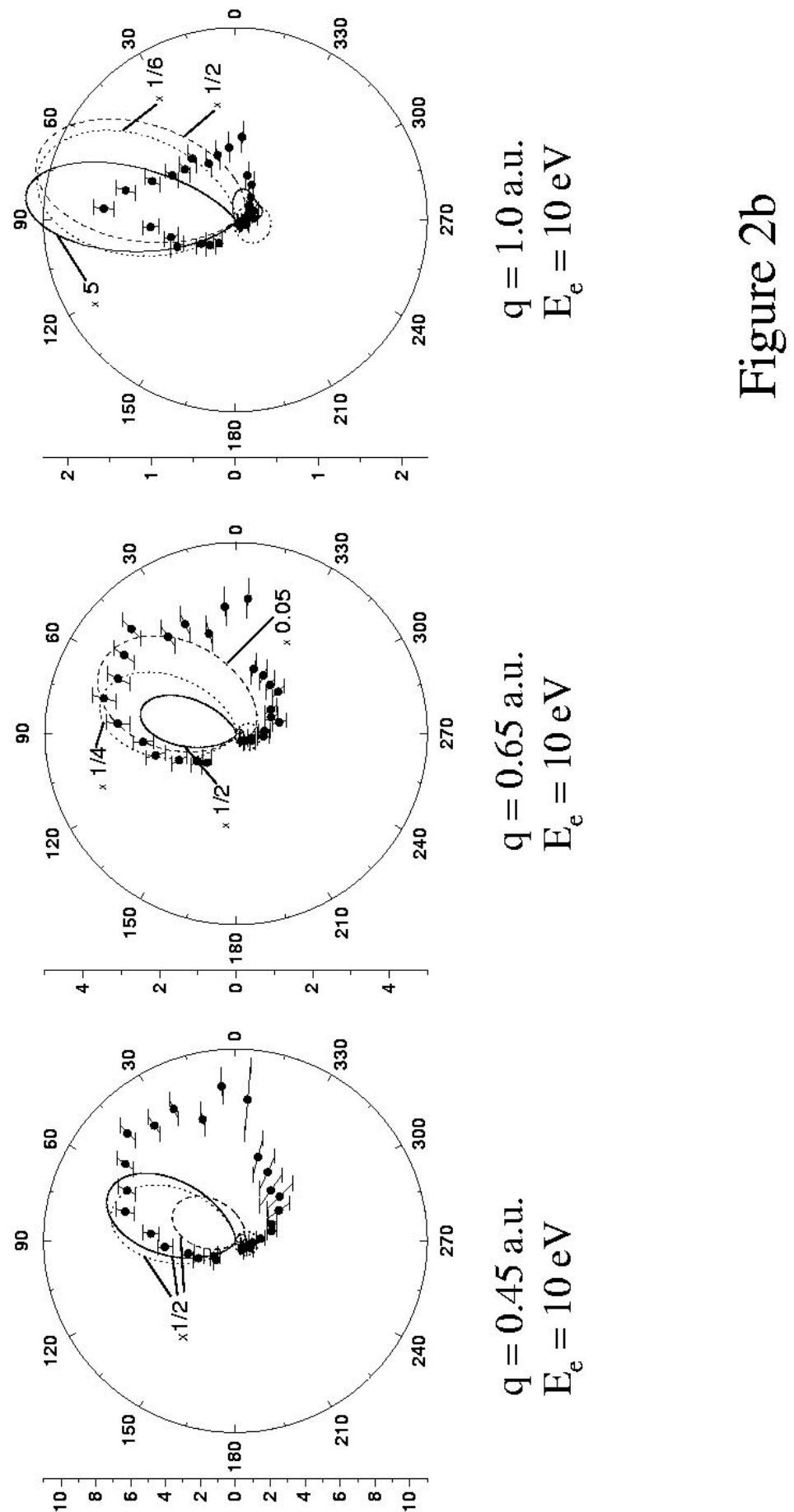

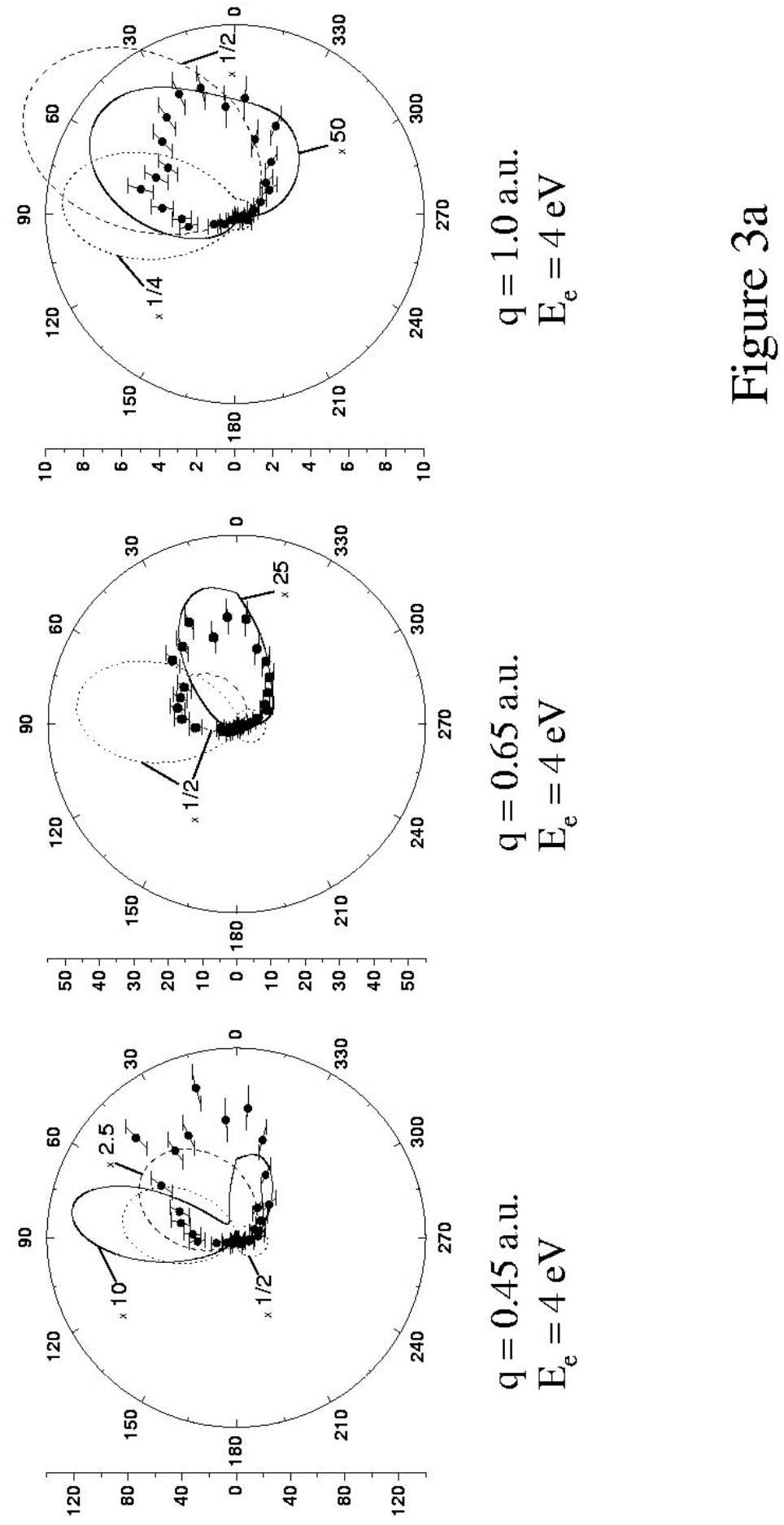

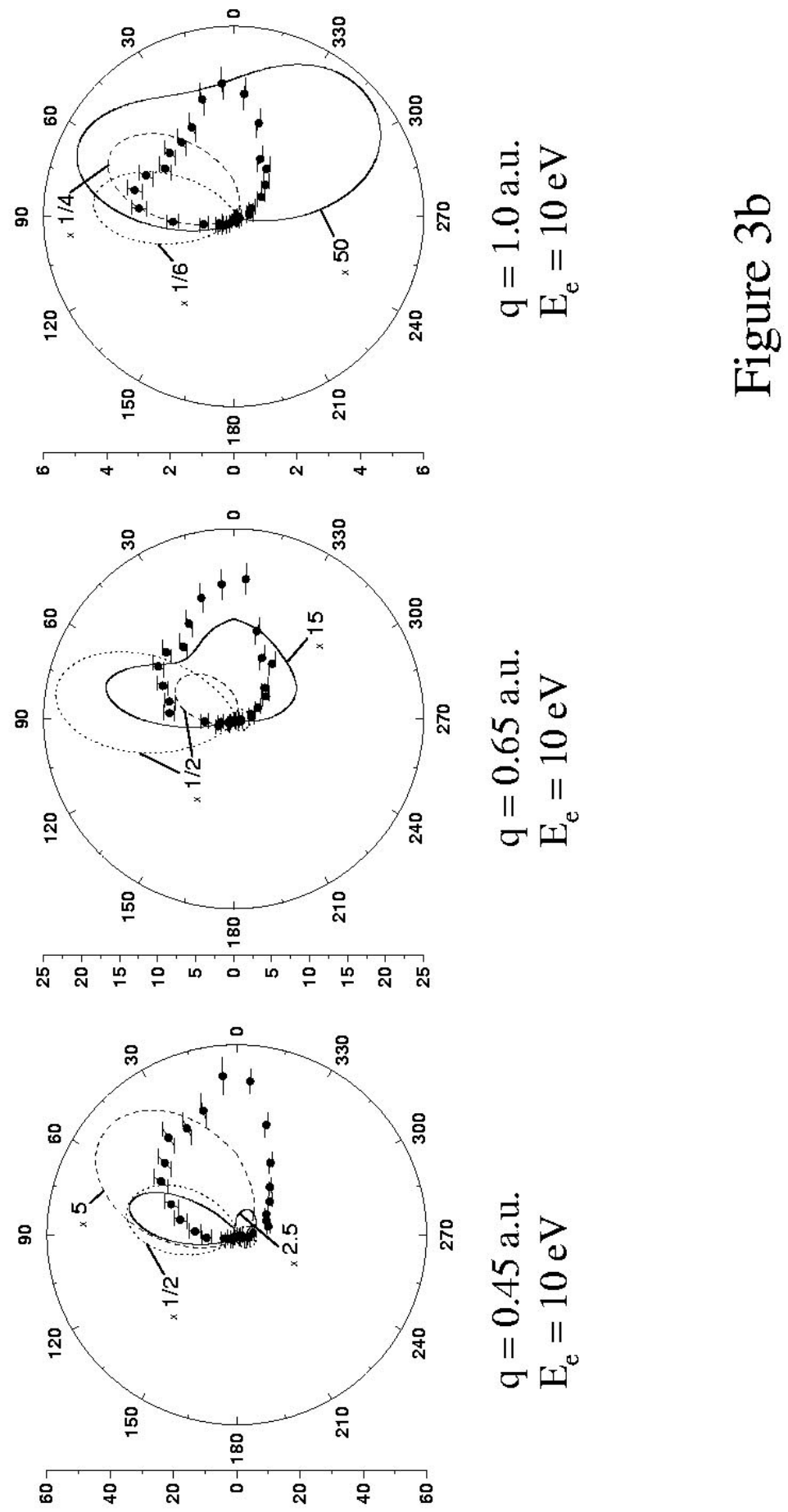

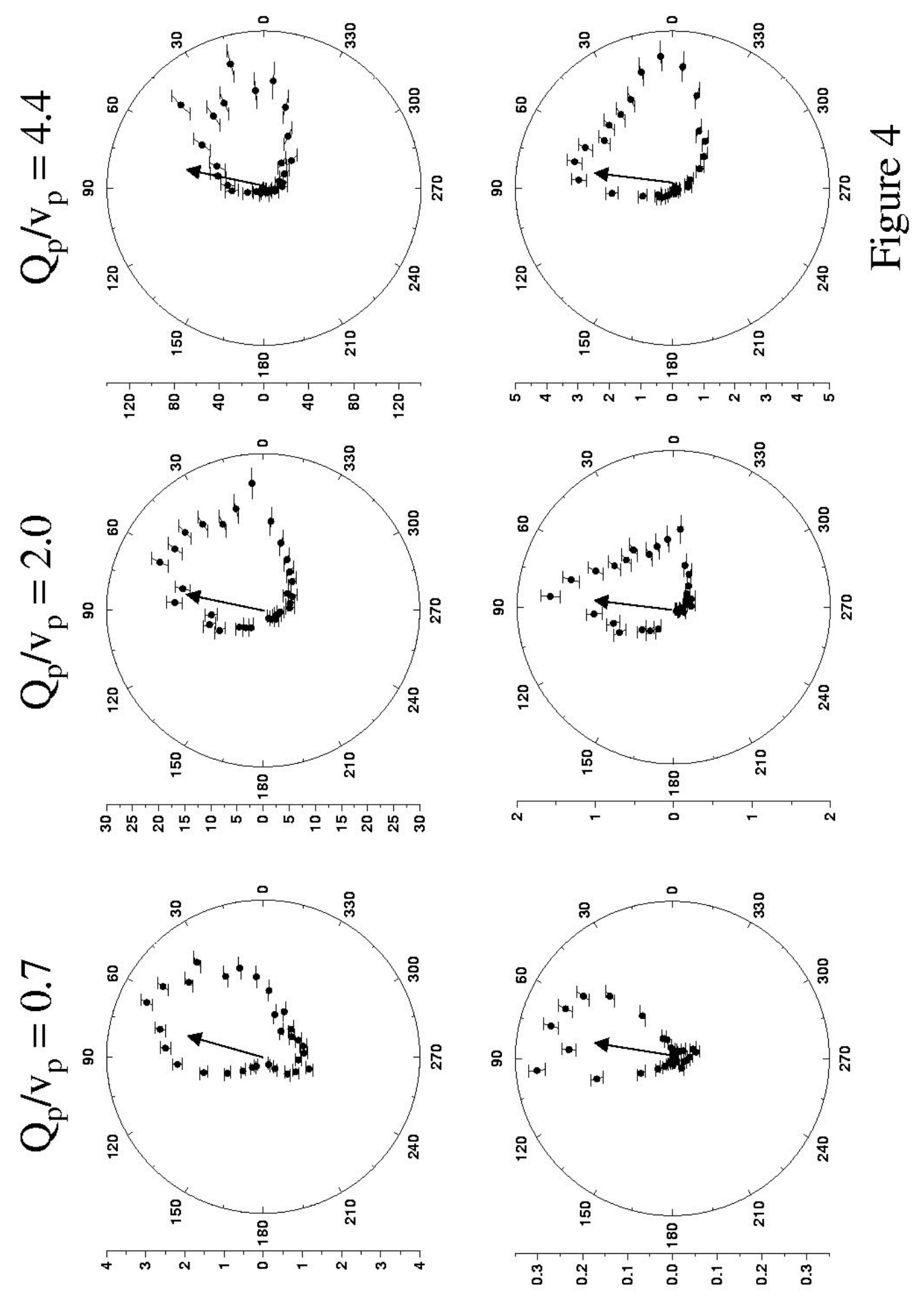

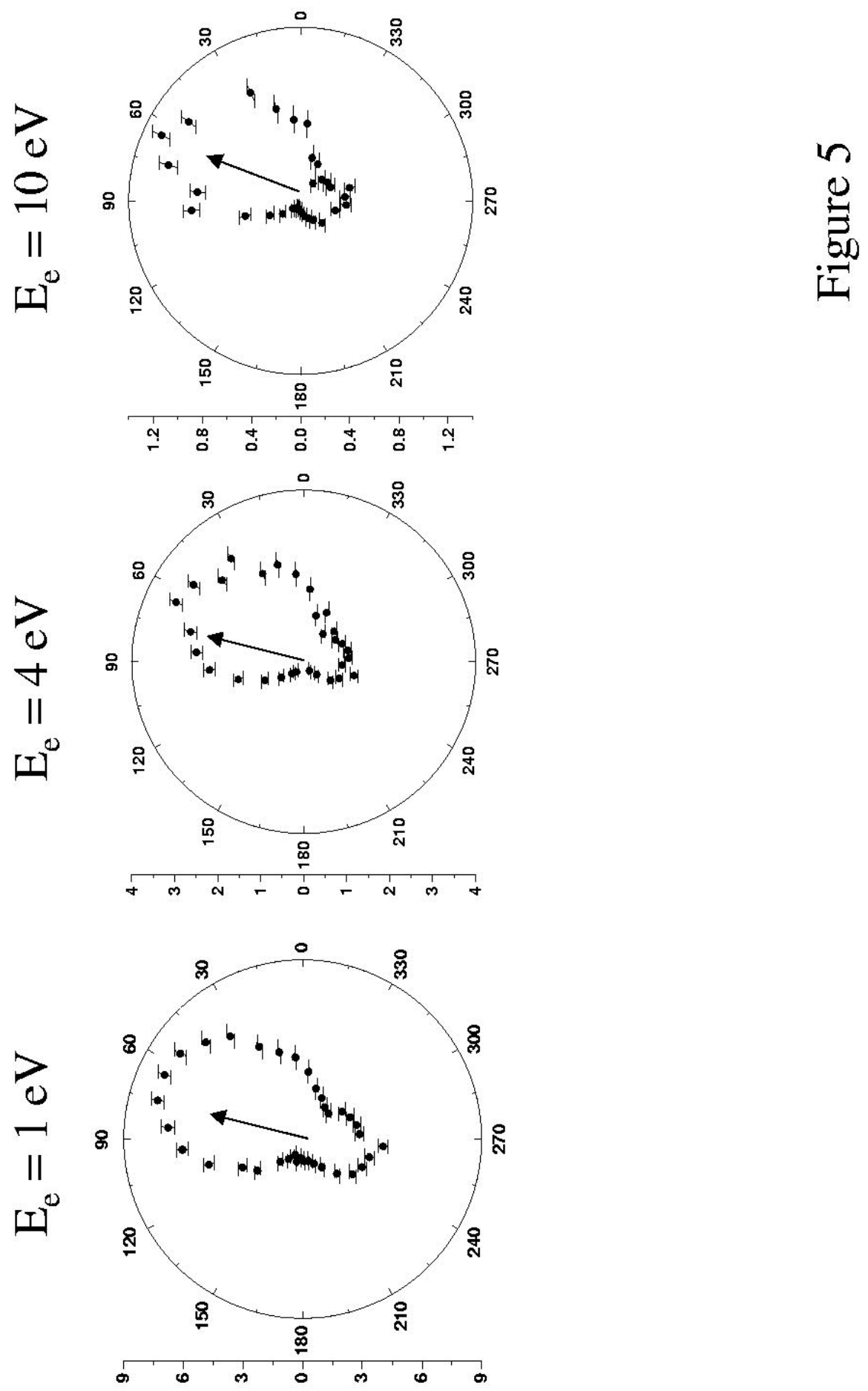
$\hat{e}$

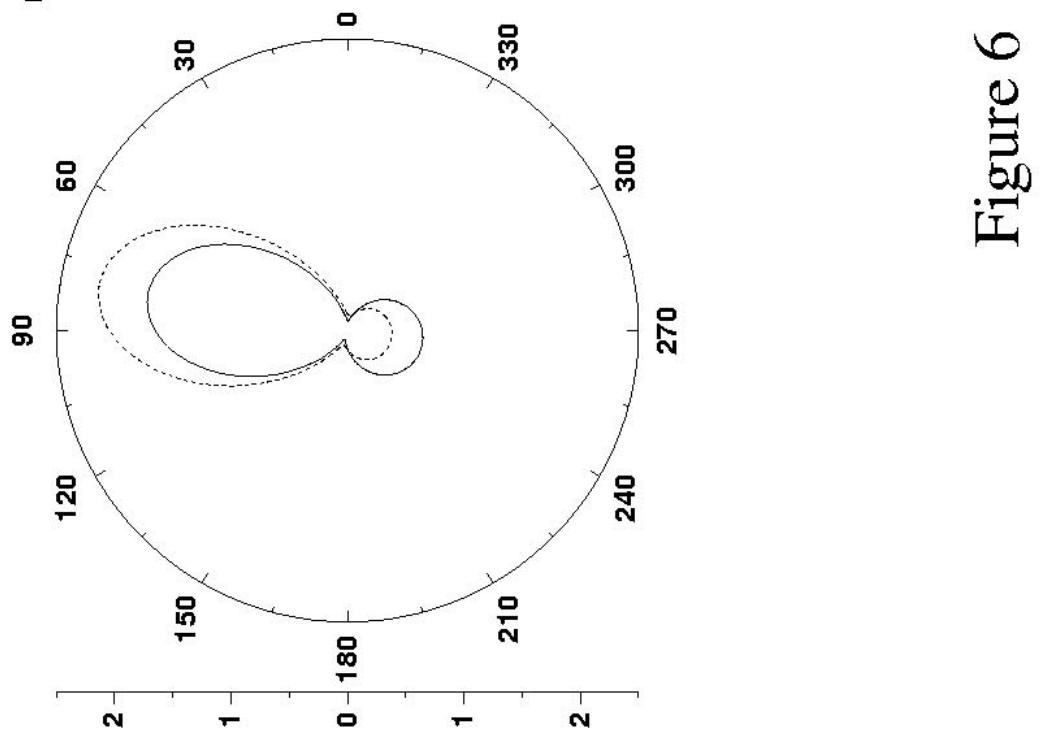

$\sqrt{8}$

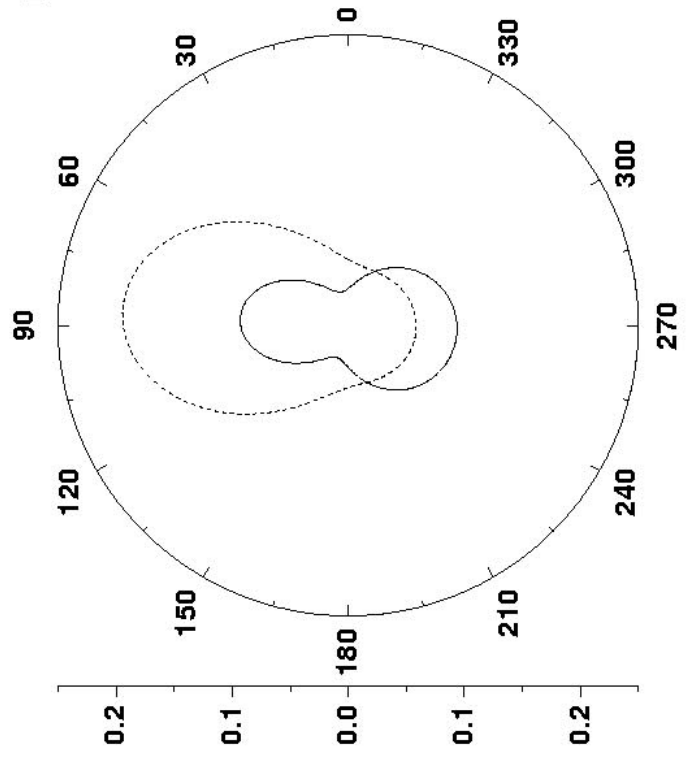

